\title{
Editors' Summary for the Special Issue: 8th Linear Algebra Workshop (LAW'17), Ljubljana, Slovenia, June 12 - 16, 2017
}

https://doi.org/10.1515/spma-2018-0031

The 8th Linear Algebra Workshop was the most recent in the series of Linear Algebra Workshops which started in 1996. Workshops are held every three years in May or June in Slovenia. The first four in 1996, 1999, 2002 and 2005 were held at Bled, next two in 2008 and 2011 in Kranjska Gora, and the last two in 2014 and 2017 were held in Ljubljana. The main theme of all workshops is the interplay between operator theory and algebra. After plenary and contributed talks in the morning, the afternoons are devoted to work in smaller groups on open questions. The 4th Linear Algebra Workshop in 2005 was organized in conjunction with the Conference in honor of Heydar Radjavi's 70th birthday, the 7th Linear Algebra Workshop in 2014 was organized in conjunction with 23rd International Workshop on Matrices and Statistics (IWMS). There were 40 to 120 participants attending each workshop.

The 8th Linear Algebra Workshop was organized by the local organizing committee: D. Kokol Bukovšek, T. Košir, M. Kramar Fijavž, the scientific organizing committee: D. Kokol Bukovšek, B. Kuzma, M. Omladič, H. Radjavi. The organizing committee chair was Heydar Radjavi, University of Waterloo, Canada. The secretary of the Workshop was Damjana Kokol Bukovšek, University of Ljubljana, Slovenia. The workshop was supported by the Institute of Mathematics, Physics and Mechanics, Ljubljana, the International Linear Algebra Society, the University of Ljubljana, Faculty of Mathematics and Physics, and the University of Primorska, Faculty of Mathematics, Natural Sciences and Information Technologies.

At the 8th Linear Algebra Workshop there were 11 plenary invited talks and 14 contributed talks. All together, 53 researchers attended it. Invited talks werte given by:

- Balint Farkas, University of Wuppertal, Germany

- Alexander Guterman, Lomonosov Moscow State University, Russia

- Dijana Ilišević, University of Zagreb, Croatia

- Thomas Laffey, University College Dublin, Ireland

- Chi-Kwong Li, College of William and Mary, USA

- Laurent Marcoux, University of Waterloo, Canada

- Mitja Mastnak, Saint Mary's University, Canada

- Vladimir Müller, Czech Academy of Sciences, Czech Republic

- Ahmed Sourour, University of Victoria, Canada

- Helena Šmigoc, University College Dublin, Ireland

- Vladimir Troitsky, University of Alberta, Canada

\footnotetext{
^Corresponding Author: Bojan Kuzma: University of Primorska, FAMNIT, Glagoljaška 8, 6000 Koper, Slovenia, E-mail: bojan.kuzma@famnit.upr.si

Damjana Kokol Bukovšek: University of Primorska, FAMNIT, Glagoljaška 8, 6000 Koper, Slovenia
}

Әopen Access. [(c) Br-Nc-ND $\odot 2018$ Damjana Kokol Bukovšek and Bojan Kuzma, published by De Gruyter. This work is licensed under the Creative Commons Attribution-NonCommercial-NoDerivs 4.0 License. 
On Tuesday, June 13, a Hans Schneider ILAS lecture was given by Vladimir Müller from the Czech Academy of Sciences, Czech Republic, who spoke on »Circles in the spectrum and numerical ranges«.

The afternoons were devoted to work in Working groups:

- Anita Buckley, Klemen Šivic: Positive maps

A linear map $\Phi: M_{n}(\mathbb{R}) \rightarrow M_{m}(\mathbb{R})$ is called $*$-linear if $\Phi\left(X^{T}\right)=\Phi(X)^{T}$ for each $X \in M_{n}(\mathbb{R})$. A $*$-linear map $\Phi: M_{n}(\mathbb{R}) \rightarrow M_{m}(\mathbb{R})$ is called positive if it maps positive semidefinite matrices to positive semidefinite matrices. Note that a $*$-linear map on the full matrix space is positive if and only if its restriction to the subspace of symmetric matrices, $\left.\Phi\right|_{\operatorname{Sym}_{n}}: \operatorname{Sym}_{n} \rightarrow \operatorname{Sym}_{m}$, is positive.

At the working group positive maps appearing in $[3,4]$ were considered. It was found that the positive maps with the maximal number of 10 zeros constructed in [2] are exactly the positive maps constructed in [3] that are extremal. Following similar methods as in [3, 4], conditions under which new families of linear maps on $3 \times 3$ matrices are positive/extremal, were investigated.

- Balint Farkas, Marjeta Kramar Fijavž: Koopman semigroups

For a complete metric space $X$ and jointly continuous semiflow $\varphi:[0, \infty) \times X \rightarrow X$, that is

$$
\varphi(t, \varphi(s, x))=\varphi(t+s, x) \quad(t, s \geq 0), \quad \text { and } \quad \varphi(0, x)=x \quad(x \in X),
$$

the Koopman semigroup $T(t): \mathbb{C}^{X} \rightarrow \mathbb{C}^{X}$ is defined as

$$
(T(t) f)(x):=f(\varphi(t, x))
$$

In the working group we have discussed possible choices of the space $X$ and possible continuity assumptions for the semigroup $T(t)$. The main goal is to characterize different properties of the flow $\varphi(t, \cdot)$ (such as continuity, invariance of closed sets or measures, minimality, positivity, order preserving, etc.) by the appropriate properties of the Koopman semigroup $T(t)$.

We have discussed the situations of

$$
T(t): C(X) \rightarrow C(X) \quad \text { and } \quad T(t): C_{b}(X) \rightarrow C_{b}(X),
$$

where $X$ is either a (locally) compact Hausdorff space, or a non-compact Banach space (or even only vector lattice), while assuming strong continuity, $\beta$-continuity or relative uniform continuity of the semigroup. We have initiated interesting discussions and opened some new topics, which may lead to some fruitful future collaboration.

- Laurent Marcoux, Heydar Radjavi: Local-to-global properties for matrix semigroups

The working group dealt with "local-to-global" properties of matrix semigroups. The common thread behind such problems may be stated thus: given a semigroup $\mathcal{S}$ of $n \times n$ complex matrices, and some local information about the semigroup, one is interested in deriving as much information as possible about the structure of the semigroup $\mathcal{S}$ itself.

Examples of what is meant by local information include:

- $\quad$ the set $\varphi(\mathcal{S}):=\{\varphi(A): A \in \mathcal{S}\}$, where $\varphi: \mathbb{M}_{n}(\mathbb{C}) \rightarrow \mathbb{C}$ is a non-zero linear functional;

- the cardinality of $\varphi(\mathcal{S})$ for a non-zero linear functional $\varphi: \mathbb{M}_{n}(\mathbb{C}) \rightarrow \mathbb{C}$; and

- the set $\mathcal{D}(\mathcal{S}):=\left\{\Delta(A):=\left(a_{k, k}\right)_{k=1}^{n}: A=\left[a_{i, j}\right] \in \mathcal{S}\right\}$ of all diagonal expectations of elements of $\mathcal{S}$.

Before citing examples from the literature, let us remind the reader that a semigroup $\mathcal{S}$ of matrices in $\mathbb{M}_{n}(\mathbb{C})$ is said to be indecomposable if it has no non-trivial standard invariant subspaces, i.e. invariant subspaces spanned by a subset of the standard basis for $\mathbb{C}^{n}$, and that $S$ is said to be irreducible if it has no non-trivial invariant subspaces at all. Also, for the purposes of this note, a matrix $A=\left[a_{i, j}\right] \in \mathbb{M}_{n}(\mathbb{C})$ is said to be non-negative if $0 \leq a_{i, j}$ for all $1 \leq i, j \leq n$.

There are a number of problems of this form which have been studied over the years and for which answers have been obtained. For example, it is known that if $\delta$ is irreducible, then $\varphi(\delta)$ is finite if and only if $\mathcal{S}$ is finite [7]. One can even find an upper bound for the size of $\mathcal{S}$, based upon the size of $\varphi(\mathcal{S})$.

As well, it was shown in [5] that if $\mathcal{S}$ is an indecomposable semigroup of non-negative matrices and $\mathcal{D}(\mathcal{S}) \subseteq$ $\prod_{k=1}^{n}\{0,1\}$, then there exists an invertible diagonal matrix $D$ such that $D^{-1} \delta D$ consists of matrices, all of whose entries lie in $\{0,1\}$. Some extensions of this result were studied in [6]. 
As yet another example, it was shown in [1] that if $\mathcal{G} \subseteq \mathbb{M}_{n}(\mathbb{C})$ is an irreducible group of matrices such that $\mathcal{D}(\mathcal{G}) \subseteq[0, \infty)$, then there exists an invertible diagonal matrix $D \in \mathbb{M}_{n}(\mathbb{C})$ such that $D^{-1} \mathcal{S} D$ consists of matrices, all of whose entries lie in $[0, \infty)$.

A number of generalizations of this last question were considered by the working group. While definitive results have yet to be obtained, partial answers were obtained for the following statements.

(a) Suppose that $\mathcal{S} \subseteq \mathbb{M}_{n}(\mathbb{C})$ is an indecomposable semigroup of positive matrices and $S_{1,1}:=\left\{a_{11}: A=\right.$ $\left.\left[a_{i j}\right] \in \mathcal{S}\right\}$ is finite. Is it true that $\mathcal{S}$ is finite?

(b) Suppose that $0<\beta<1$, and that $\mathcal{S}$ is an indecomposable semigroup of non-negative matrices, whose diagonal entries all lie in $\{\beta, 1\}$. Does there exist an invertible diagonal matrix $D$ such that $D^{-1} \mathcal{S} D$ has entries in a prescribed finite set?

(c) The following theorem was obtained:

Theorem. Suppose that $\mathcal{S} \subseteq \mathbb{M}_{n}(\mathbb{C})$ is an indecomposable semigroup of non-negative matrices of rank at most 1, and that there exists a group $G \subseteq \mathbb{R}^{+}$such that the diagonal entries of every element of $\mathcal{S}$ lie in $P:=(G \cap[0,1]) \cup\{0\}$. Then there exists an invertible diagonal matrix $D$ such that $D^{-1} \mathcal{S} \subseteq \mathbb{M}_{n}(P)$.

To place things in context, it was noted that there exists an indecomposable semigroup of matrices such that all diagonal entries of all elements of the semigroup are non-negative, and yet no diagonal similarity will make all entries of all matrices non-negative.

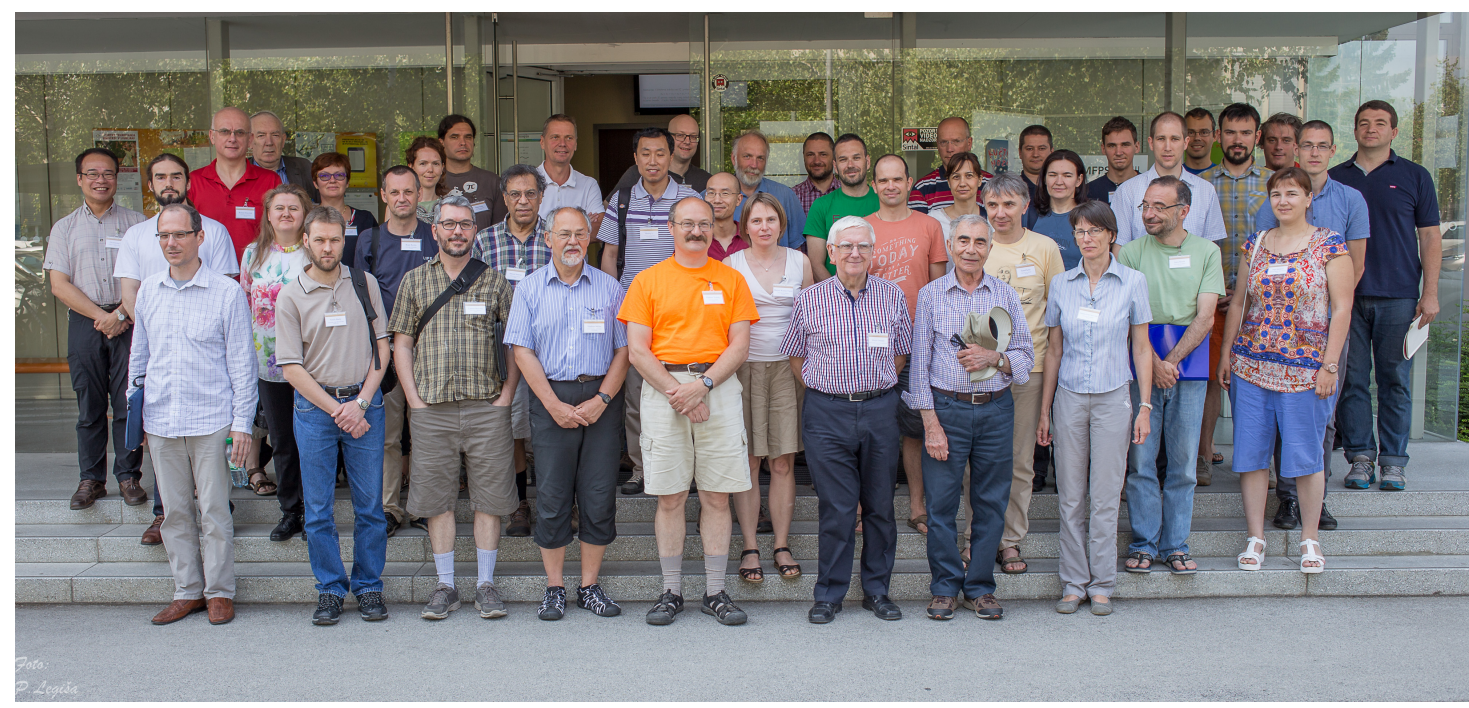

To see a list of participants, abstracts of the talks and working groups and some photos, please visit http://www.law05.si/.

\section{References}

[1] J. Bernik, M. Mastnak, H. Radjavi, Positivity and matrix semigroups, Linear Algebra Appl. 434 (2011), 801-812.

[2] A. Buckley, K. Šivic, Nonnegative biquadratic forms with maximal number of zeros, preprint, available at https://arxiv.org/ pdf/1611.09513.pdf.

[3] S. J Cho, S. H. Kye, S. G. Lee, Generalized Choi Maps in Three-Dimensional Matrix Algebra, Linear Algebra Appl. 171 (1992), 213-224.

[4] J. Hou, C. K. Li, Y. T. Poon, X. Qi, N. S. Sze, A new criterion and a special class of k-positive maps, Linear Algebra Appl. 470 (2015), 51-69.

[5] L. Livshits, G. MacDonald, H. Radjavi, Positive matrix semigroups with binary diagonals, Positivity, 15 (2011) 411-440.

[6] A. Popov, H. Radjavi, P. Williamson, Nonnegative matrix semigroups with finite diagonals, Linear Algebra Appl. 434 (2011), 1409-1424.

[7] H. Radjavi, P. Rosenthal, Limitations on the size of semigroups of matrices, Semigroup Forum, 76 (2008), 25-31. 